\title{
Modeling and Optimization of RF-Energy Harvesting-assisted Quantum Battery System
}

\author{
Sumit Gautam, Shree K. Sharma, Symeon Chatzinotas, and Björn Ottersten \\ Interdisciplinary Centre for Security, Reliability and Trust (SnT), University of Luxembourg, Luxembourg. \\ Email: \{sumit.gautam,shree.sharma,symeon.chatzinotas,bjorn.ottersten\}@uni.lu
}

\begin{abstract}
The quest for finding a small-sized energy supply to run the small-scale wireless gadgets, with almost an infinite lifetime, has intrigued humankind since past several decades. In this context, the concept of Quantum batteries has come into limelight more recently to serve the purpose. However, the main issue revolving around the closed-system design of Quantum batteries is to ensure a loss-less environment, which is extremely difficult to realize in practice. In this paper, we present the modeling and optimization aspects of a Radio-Frequency (RF) Energy Harvesting (EH) assisted Quantum battery, wherein several EH modules (in the form of micro- or nano- sized integrated circuits (ICs)) help each of the involved Quantum sources achieve the so-called quasi-stable state. Specifically, a micro-controller manages the overall harvested energy from the RF-EH ICs and a photon emitting device, such that the emitted photons are absorbed by the electrons in the Quantum sources. In order to precisely model and optimize the considered framework, we formulate a transmit power minimization problem for an RFbased wireless system to optimize the number of RF-EH ICs under the given EH constraints at the Quantum battery-enabled wireless device. We obtain an analytical solution to the abovementioned problem using a rational approach, while additionally seeking another solution obtained via a non-linear program solver. The effectiveness of the proposed technique is reported in the form of numerical results by taking a range of system parameters into account.
\end{abstract}

Index Terms-Quantum battery, RF-Energy Harvesting, transmit power optimization.

\section{INTRODUCTION}

The technological advancements in the field of Wireless Communications continue to amaze the mankind in several ways. In this direction, the journey from the binary information bits to the Quantum bits (Qubits) seems to be approaching its destination faster than expected [1]. With the exiting methodologies inching closer to the Quantum-based techniques day-by-day, it becomes essential to seek the possibility of establishing a technically synergistic relationship between the Quantum theories and Wireless Communications-based techniques [2], [3]. However, a major hurdle in this progress is our dependence on the energy sources, which are as strong as on the gadgets themselves [4]-[6].

In order to address these challenges, the idea of Quantum batteries has been put forward by various researchers [7][10]. In this vein, a recently proposed idea of a nano-meter sized Quantum battery shows great promise [11]. Similar to the phenomenon of Luminescence, a nano-meter sized Quantum battery composed of nanostructured solid-state of matter works on the principle that electrons can absorb photons and stay in the energized state for possibly an infinite time-period. However, this possibility is theoretically feasible within a loss-less closed-system, often leading to a quasistable state. It is noteworthy that this phenomenon remains the backbone of several closed-system type of Quantum batteries [12]. Ensuring this condition is difficult in practice, but a mechanism could be sought wherein an radio-frequency (RF)based wireless energy harvesting (EH) method is utilized.

An early EH concept of getting energy from/through the air to power all equipment, was proposed by Nikola Tesla in the late 1890s to early 1900s [13], [14]. Ever since then, this concept has found its applications in several fields of research including vehicles [15], wireless gadgets [16], flying objects [17], etc. More recently, Varshney discussed about the possibility of using RF based simultaneous wireless information and energy transmission (SWIPT) [18], which involves the traditional information receiver unit, along with an EH module. In this context, several receiver architectures for SWIPT have been proposed in the literature based on timeswitching (TS), power-spliting (PS), or separated architecture (SA) [19]. The RF-based EH module is comprised by the concatenation of an RF-antenna, matching circuit, rectifier circuit and a polarized capacitor, thereby making it a passive device [20]. The EH module usually suffers due to the saturation effect at the involved diode element. Correspondingly, the non-linear nature of EH operation is observed due to the saturation effect. However, this effect may be rectified by placing several EH circuits in parallel, yielding a sufficiently large linear conversion region in practice [21], [22]. The RF$\mathrm{EH}$ mechanism is also considered as an upcoming frontier in establishing green wireless communication systems [23].

There are several works which have investigated the EH possibilities at a device via ambient sources, such as thermal, solar, mechanical, etc., to assist the Quantum batteries [24]. However, the consideration of Quantum batteries with RFEH mechanism is still an unexplored and open area of research. In the current scenario, realizing a system which incorporates a Quantum battery and EH modules is difficult, primarily due to the large size of the EH module. Moreover in the future, the authors envision that the size of the $\mathrm{EH}$ modules may be shrunk tremendously into micro- to nanometer sized integrated circuits (ICs), which may be utilized to assist Quantum batteries in some way. The Quantum batteries requires assistance from the external sources so as to compensate for the losses that may be incurred due to internal operations, while maintaining their stability. Consequently, the consideration of novel concept aiming at the modeling and optimization of a design with a set of RF-EH ICs placed in parallel to assist a Quantum battery seems promising. 


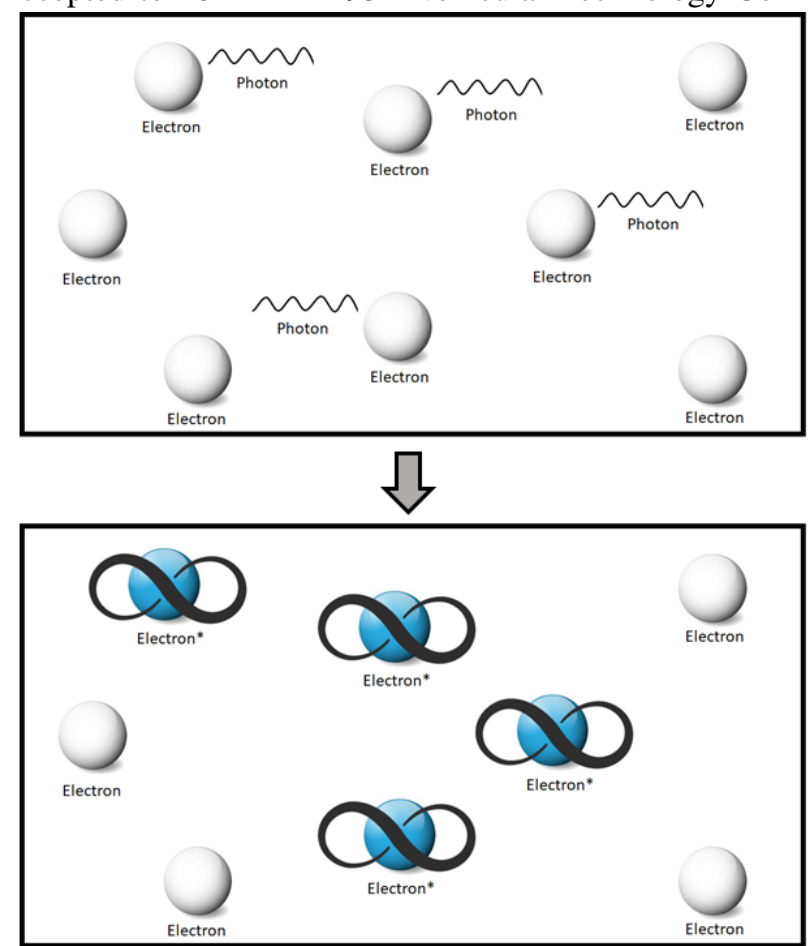

Fig. 1: The phenomenon of absorption of photons by electrons in nanostructured solid state of matter within a closed-system, where they may theoretically stay in this condition for nearly infinite time.

In this paper, we present a novel framework with the consideration of several RF-EH ICs assisting an end-user equipped with a Quantum battery. A number of RF-EH ICs collect energy from RF signals transmitted from the transmitter. The collected energy from all the RF-EH ICs is then managed by a micro-controller, which consequently operates a photon emitting device. The photon emitting device is responsible for the transfer of energy from the photon to an electron within the Quantum sources present within the Quantum battery. In this context, we formulate the optimization problem to minimize the overall transmitted power, subject to limitations on the overall harvested energy at the end-user node, and the number of RF-EH ICs. We investigate the problem in two ways. First, we analyze the problem using theoretical rigour to obtain the closed form solutions for the intended parameters. Second, we directly solve the (main) formulated non-convex problem using an optimization solver. Specifically, the main contributions and novelty of this work are summarized below.

(a) We present a novel design of a receiver device equipped with RF-EH ICs assisted Quantum battery. In the proposed design, a Quantum battery is present at the core of the end-user device and is assisted via set of RF-EH ICs.

(b) To model and optimize the above-mentioned novel scheme, we formulate an optimization problem to minimize the overall transmitted power, while ensuring that the energy requirements at the end-users are successfully met. This process is performed in conjugation with the estimation of the number of RF-EH ICs, in order to meet the requirements of the system design.

(c) We present optimal solutions to the above-mentioned

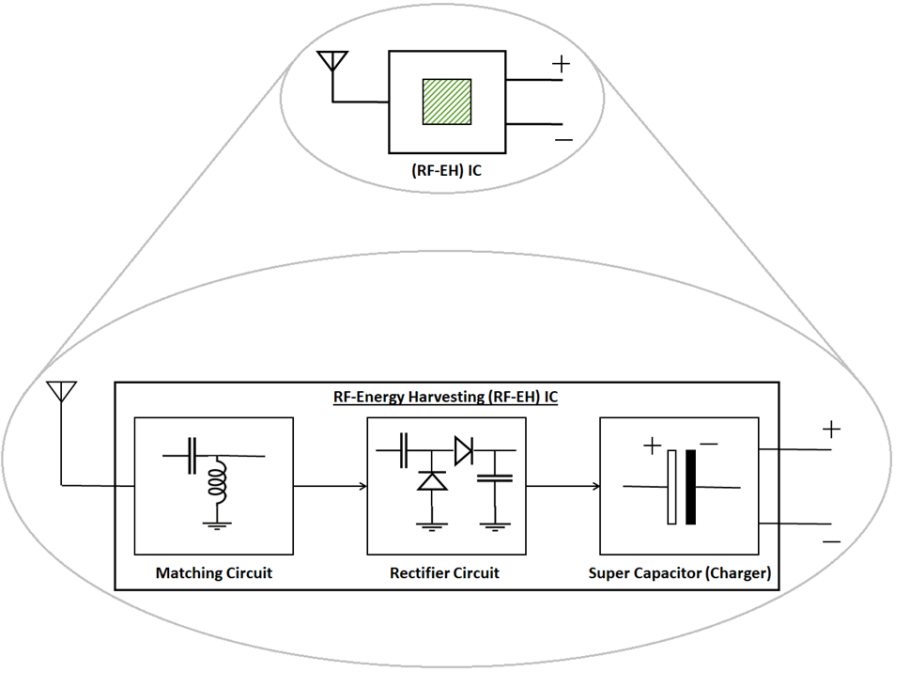

Fig. 2: Depiction of the proposed RF-EH IC comprising a microor nano- sized passive RF-EH circuit. Ideally, an RF-antenna traps the ambient (or specific band(s)) of RF-signal and converts it to equivalent DC-type of output.

problem. We first find closed-form solutions using theoretical analysis and then validate the results by directly solving the main problem via a non-convex optimization solver.

(d) We show the effectiveness of the proposed methods for design and optimization of the considered framework, under several operating parameter values and conditions. The presented results motivate towards the practical application of the proposed futuristic design.

Further sections of this paper are organized as follows. Section II provides an introduction to the system model. The proposed RF-EH-assisted Quantum Battery System design and the problem of transmit power minimization are both presented in Section III. Numerical results are shown in Section IV, followed by concluding remarks in Section V.

\section{SYSTEM MODEL}

In this section, we discuss the operation of RF-EH ICs assisted Quantum batteries along with its possible future prototype. To proceed, we discuss some key aspects involved in the system design, to provide motivation for our proposed framework. A variety of Quantum battery concepts have been proposed in the literature [7]-[10], while considering the possibilities of closed- and open-system designs. Evidently, there are a wide range of challenges in the open-system designs of the Quantum batteries [12]. Therefore, we consider the closed-system design for our analysis.

Pertaining the closed-system design, the concept of Quantum batteries broadly works on the principle that an electron absorbs a photon to reach to an excited state using its energy, and may remain in the same condition for almost an infinite time. This phenomenon finds its application in the conceptual nano-meter sized Quantum batteries [11], which are capable of holding the electric charge for an infinite time-period. An illustration of this mechanism is projected as in Fig. 1. The process is similar to the Luminescence effect, when certain objects, e.g., dials of watches, plastic figures, etc., absorb photons and glow in the dark for a very long time. 


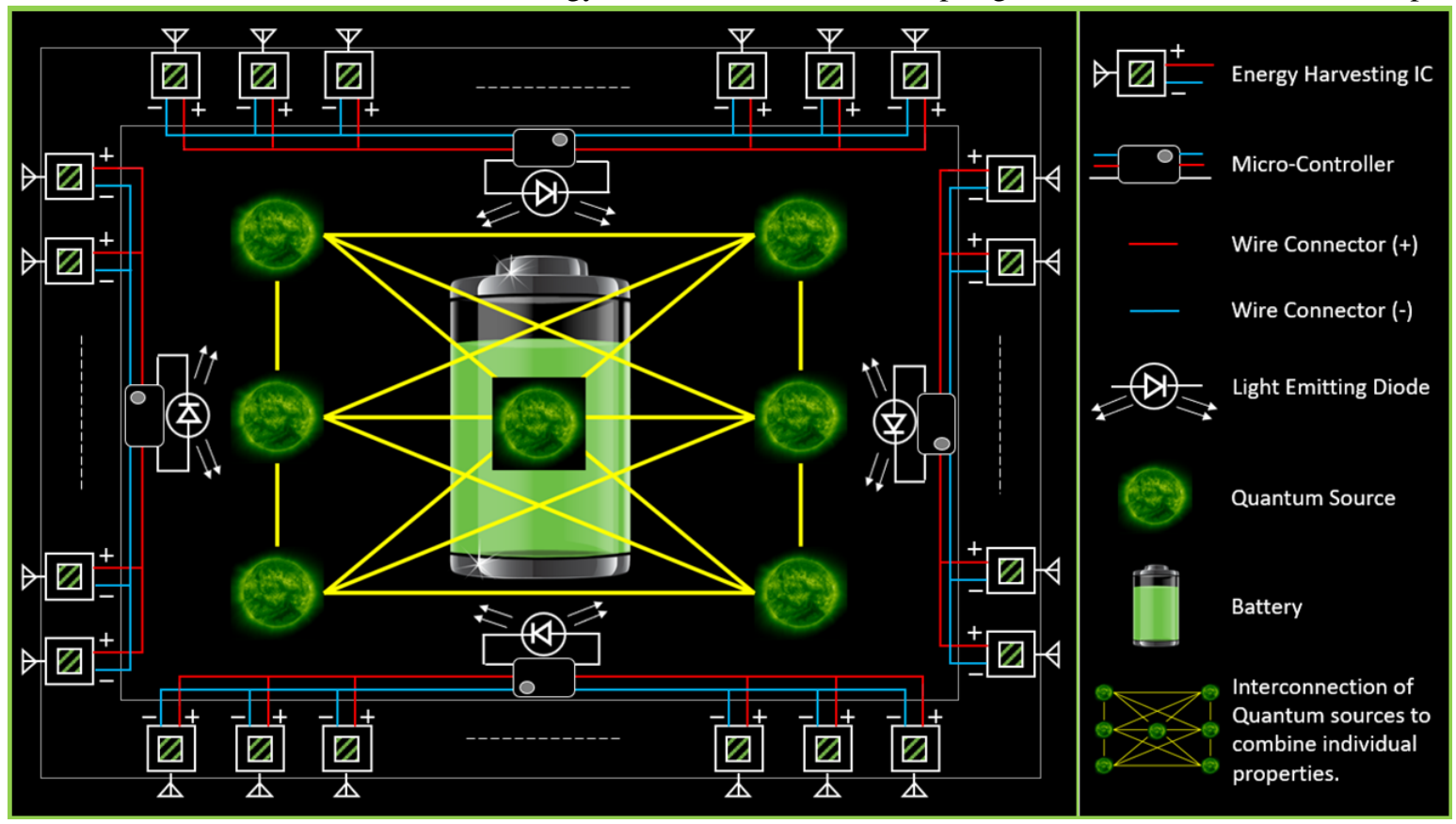

Fig. 3: Schematic of the proposed RF-EH ICs assisted Quantum battery.

The above-mentioned process may seem sound from a theoretical view-point. However, its realization in practice is very difficult due to the presence of a variety of impacting parameters, such as environment, heat losses, component functioning losses, etc. In this regard, ensuring a completely loss-less environment is cumbersome and almost impractical, given the current state of technology. Correspondingly, there have been several designs of Quantum batteries leveraging from the harvested energy via ambient sources, such as solar, thermal, mechanical, etc., [24].

Another potential technique for harvesting energy from the RF-based signals has been developed recently to cater to the energy needs of the wireless devices. A basic EH module is comprised of passive elements-based matching circuit, rectifier circuit and a charge holding capacitor element. The EH receivers are available in the form of general device-sized modules. Similar to the evolution of RF-based information receivers, we assume that the EH circuits will be miniaturized in future, and can be made available in the form of RF-EH ICs, as shown in Fig. 2.

In this direction, we consider that a wireless RF-transmitter emits the signal $x(t)$ intended for the RF-EH IC(s) via a single antenna. The corresponding EH signal waveforms may be designed according to the frameworks proposed in [25]. Noticeably, there is an availability of a wide range of suggestions on the consideration of waveform designs for $\mathrm{EH}$ applications in the literature. Thus, it is difficult to reach to a mutual consensus in terms of efficient waveform schemes, since their effectiveness is largely dependent on the type of EH circuit used. Therefore, we assume a generalized zero mean and unit variance signal in this paper for ensuring a theoretical tractability.

The overall transmit power is considered as $P_{T}$. The Quantum battery equipped end-user receives the signal: $y(t)=$ $h(t) * x(t)+n_{R}(t)$, where $h(t)$ is the complex channel gain coefficient for the wireless channel and $n_{R}(t)$ is the additive zero mean Gaussian noise at the corresponding end-user's receiving antenna equipment with a noise variance of $\sigma_{R}^{2}$. Therefore, the linear EH operation at the RF-EH IC unit of the receiver is given as: $\mathcal{E}^{\mathcal{L}}=\zeta_{i}\left(P_{T}|h|^{2}+\sigma_{R}^{2}\right)$, where $0<\zeta_{i} \leq 1$ is the energy conversion efficiency of the corresponding receiver. Noticeably, $\mathcal{E}^{\mathcal{L}}$ is theoretically valid for numerical evaluations, however, its practical implementation is questionable. This calls for the adoption of a sigmoidal function based non-linear EH model [26]-[28], defined as

$$
\mathcal{E}^{\mathcal{N}}=\frac{\mathcal{E}^{\prime}}{1-\phi} \cdot\left(\frac{1}{1+e^{\left(-\alpha P_{T}|h|^{2}+\alpha \beta\right)}}-\phi\right),
$$

where $\phi \triangleq \frac{1}{1+\exp (\alpha \beta)}$, the constant $\mathcal{E}^{\prime}$ is obtained by determining the maximum harvested energy on the saturation of the EH circuit, and $\alpha$ and $\beta$ are specific to the capacitor and diode turn-on voltage metrics at the EH circuit. Further, we assume normalized time slots to use the terms power and energy interchangeably.

\section{Proposed Design of RF-EH-ASSisted QuANTUM BATTERY SYSTEM}

Based on the aforementioned concepts, we now present the proposed novel design of RF-EH ICs assisted Quantum battery. The basic system schematic of the proposed framework is shown in Fig. 3, which consists of the Quantum battery at the core of the receive unit. In order to compensate for the losses that may have incurred during the device operation, the device reports to the nearest transmit source, which can be through regular RF-based wireless communications method or via Quantum-based wireless communications systems using entanglement of Qubits [2], [3]. The transmitter then emits an RF-signal for transferring energy to the multiple RF-EH ICs 
present all around the receiver. ${ }^{1}$ We assume the availability of $n$ number of RF-EH ICs, with a maximum cap of $N$. The selection of $N$ is naturally decided according to the design requirements, e.g., the size of the device.

The received signal at the RF-EH ICs is converted into electrical energy using the internal passive circuit, while a microcontroller is responsible for the management of the overall harvested energy. In order to facilitate an energy exchange process to the Quantum battery core, a very low intensity photon-emitting device (e.g., light emitting diode (LED)) is attached to the micro-controller, which is responsible for its operation as well. Let us consider that $\xi=\eta+\kappa$ is the overall harvested energy demand at the end-user. Correspondingly, ' $\eta$ ' accounts for the harvested energy demand to enable the photon emission. Whereas ' $~ \kappa$ ' is the additional harvested energy demand required for other consumption metrics, such as operation of micro-controller, compensation for losses, and maintaining suitable temperature within the closed-system.

To proceed, we intend to model and optimize the involved metrics and parameters in the considered framework. Specifically, we develop the formulation to minimize the overall transmitted power under limitations on the minimum harvested energy and the number of RF-EH ICs. The succeeding section provides more insight on the considered problem and the obtained (proposed) solutions.

\section{A. Problem Formulation and Proposed Solution}

Herein, we formulate the optimization problem to minimize the total transmit power, subject to the constraints on the harvested energy by RF-EH ICs, and the number of (nonlinear) EH ICs. The corresponding optimization problem can subsequently be written in its mathematical form as follows

$$
\begin{aligned}
(P 1): \min _{n, P_{T}} & P_{T} \\
\text { s.t. } & (C 1): n \mathcal{E}^{\mathcal{N}} \geq \xi, \\
& (C 2): n \leq N .
\end{aligned}
$$

It is clear that $(P 1)$ is a non-convex problem, primarily due to the joint optimization of $n$ and $P_{T}$ in (3). However, an analytical solution based on graphical/direct-evaluation method is possible. In this regard, we first observe that the objective in $(P 1)$ is independent of $n$. Next, we find that $\mathcal{E}^{\mathcal{N}}$ is an increasing function of $P_{T}$, while the presence of $n$ indicates that the constraint may be satisfied by either increasing its value or the value of $P_{T}$. More specifically, keeping the value of $n=N$ would ensure an optimized solution of $P_{T}$, implying that the equality in (4) must hold. Therefore, by leveraging from one solution, i.e., $n=N$, we reach to the following (reduced) problem.

$$
\begin{aligned}
(P 2): & \min _{P_{T}} \\
\text { s.t. } & (C 1): N \mathcal{E}^{\mathcal{N}} \geq \xi .
\end{aligned}
$$

We now have a convex problem in the form of $(P 2)$, which can either yield a closed-form solution or may be solved via

\footnotetext{
${ }^{1}$ The assumption of multiple RF-EH ICs is due to the saturation effect of the diode, which prohibits a single unit of RF-EH IC from harvesting energy beyond a certain limit.
}

well known convex optimization techniques [29]. In order to obtain a closed-form solution for $P_{T}$, we observe that the optimum solution is obtained when the equality in (6) holds. This is based on the fact that minimal transmit power will be utilized for sufficing the harvested energy demand at the enduser, while taking into consideration that all the $N$ RF-EH ICs are active. Hence, we reach to the following solution of $P_{T}$, based on the above-mentioned analysis. ${ }^{2}$

$$
P_{T}=\frac{1}{\alpha|h|^{2}}\left(\alpha \beta-\ln \left(\frac{N \mathcal{E}^{\prime}-\xi(1-\phi)-N \phi \mathcal{E}^{\prime}}{\xi(1-\phi)+N \phi \mathcal{E}^{\prime}}\right)\right) \text {. }
$$

We note that $N$ plays a crucial role in the system design. An intuitive interpretation from the solution provided in (7) would be that the higher the number of RF-EH ICs in the device, the lower will be the transmitted power. As discussed before, the micro-controller can then manage the harvested energy to cater to the various needs to maintain the stability of Quantum battery. In the subsequent section, we present some simulation results to test the effectiveness of the proposed design in terms of the obtained solutions.

\section{Simulation Results}

In this section, we present the numerical results to evaluate the performance of the proposed system. The proposed solutions are analyzed using the MATLAB R2019b, with optimization performed via fmincon $(\cdot)$ solver present in the optimization toolbox [30], where the solutions are obtained with the help of interior point algorithm [29]. We hereby refer to these results as 'Simulation'. The closed-form solutions obtained in (7) are implemented directly via MATLAB R2019b, termed as 'Theoretical' for further reference.

We assume an ITU-R outdoor framework (site-general model for propagation within street canyons) [31] to generate channel realizations with the path-loss exponent:

$$
P L(D, F)=10 a \log _{10}(D)+b+10 c \log _{10}(F)+N(0, \sigma) \mathrm{dB},
$$

where $D$ is $3 \mathrm{D}$ direct distance between the transmitting and receiving stations $(\mathrm{m}), F$ is the operating frequency $(\mathrm{GHz})$, the coefficients $a, b$, and $c$ are associated with the increase of the path loss with distance, the offset value of the path loss, and the increase of the path loss with frequency, respectively, and $N(0, \sigma)$ is a zero mean Gaussian random variable with a standard deviation $\sigma(\mathrm{dB})$. The channels coefficients for the link between the transmitter and receiver are generated accordingly. Specifically, we choose: $F=24 \mathrm{GHz}, D$ is assumed to be $5 \mathrm{~m}$ (unless specified otherwise) for the enduser with respect to the transmitter, $a=2.12, b=29.2, c=$ 2.11 and $\sigma=5.06 \mathrm{~dB}$. The constants corresponding to the non-linear EH circuit are chosen as $\mathcal{E}^{\prime}=2.8 m \mathrm{~J}, \alpha=1500$, and $\beta=0.0022$ [26]-[28]. An average of 500 random channel realizations is presented for each experiment. Furthermore, we refer to the closed-form solution as theoretical and the fmincon(.) solver yielded solutions as the simulation results.

Fig. 4 shows the variation in minimized transmit power values with increasing demand of harvested energy at the end user. Herein, we compare the performances of the proposed

\footnotetext{
${ }^{2}$ Please refer to Appendix A for detailed solution.
} 
Accepted to 2021 IEEE 93 ${ }^{\text {rd }}$ Vehicular Technology Conference: VTC2021-Spring, Helsinki, Finland, 25 - 28 April 2021.

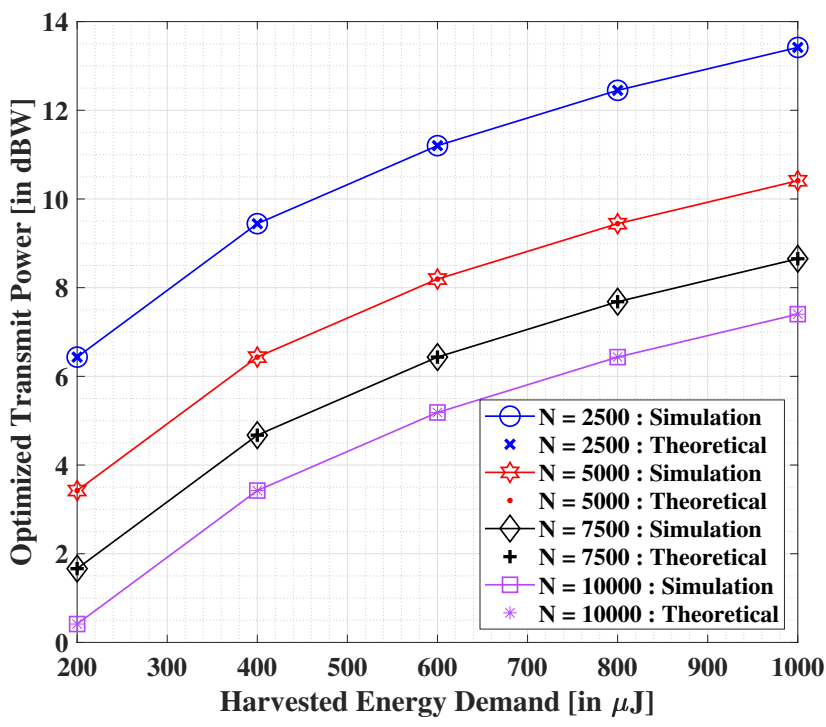

Fig. 4: Optimized transmit power versus the harvested energy demand for various values of $N$.

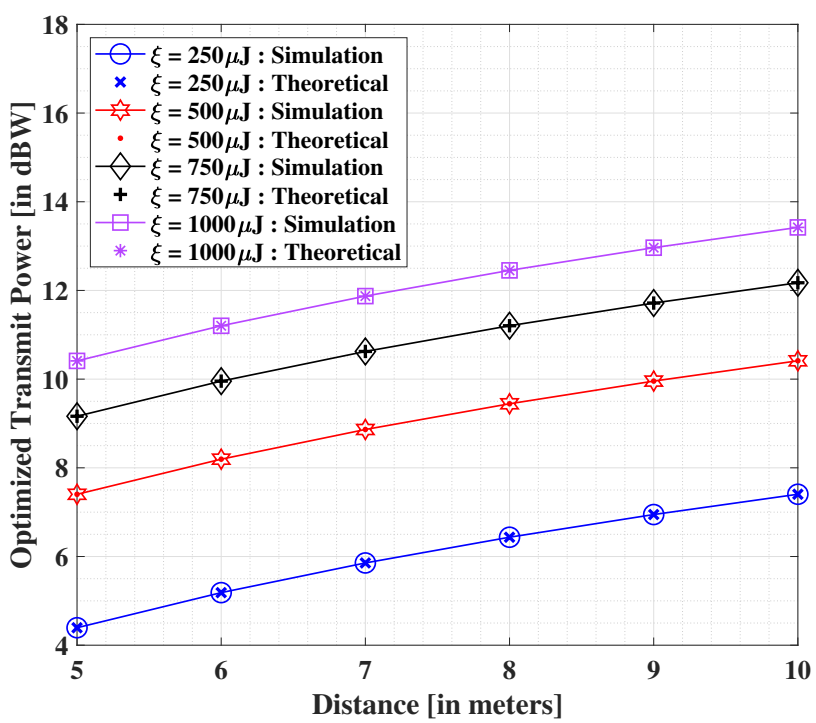

Fig. 5: Optimized transmit power versus the distance for various values of harvested energy demand, where $N=5000$.

theoretical and simulation based solutions. We observe that the optimized transmit power increases non-linearly with increasing demand of harvested energy. However, a significant decrement in the values of optimized transmit power is seen with the increasing number of RF-EH ICs. We can note that both theoretical and simulation based solutions yields the same results. It is noteworthy that these results are in-line with our intuitive analysis.

In Fig. 5, we illustrate the effect on the optimized transmit power values with the increase in the distance between the transmitter and the end-user, where $N=5000$. Herein, we draw a comparison between the theoretical and simulation based solutions, wherein both the solutions are reported to be identical. We observe that the minimum optimized transmit power increases with increasing distance values between the transmitter and end-user. However, this effect amplifies at a

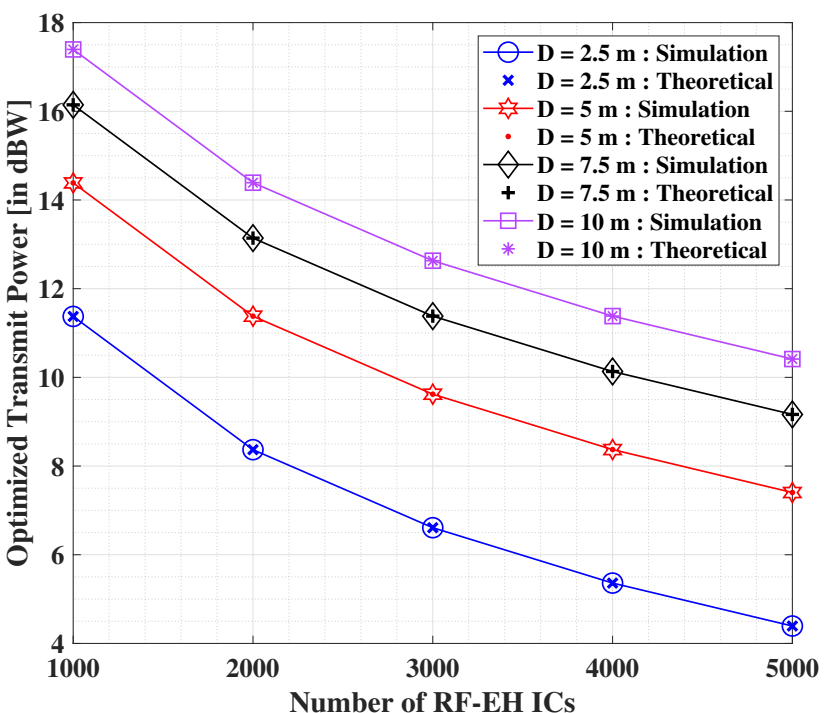

Fig. 6: Optimized transmit power versus $N$ for various values of separation distance between the transmitter and end-user.

higher level with the increasing demands of harvested energy. The clear impact of distance limitation may be inferred herein.

We show in Fig. 6 the impact of increasing the number of RF-EH ICs on the optimized transmit power values, with the assumption of $\xi=500 \mu \mathrm{J}$. Both the theoretical and simulation based solutions are found to be identical. We observe that the minimum optimized transmit power decreases with the increasing values of $N$. We additionally observe that the minimum transmit power increases with the increase in distance between the transmitter and the end-user. This outcome suggest that the more closer the end-user is to the transmitter, better will be the transmit power optimization. Additionally, if the number of RF-EH ICs are large enough, the transmit power can be further minimized drastically.

\section{CONCLUSion}

We proposed a novel design of RF-EH ICs assisted Quantum battery system, wherein several RF-EH ICs are connected in parallel, and the overall harvested energy can be managed by a micro-controller, which is responsible for powering a photon emitting device (such as an LED). With the assumption of a wireless system comprising a single transmitter and a single Quantum battery-enabled user, we formulated an optimization problem to minimize the total transmit power as well as the number of RF-EH ICs, subjected to the constraints on the harvested energy demands. A closed-form solution was obtained, along with the additional validation via a nonconvex optimization solver. For future practical systems, the outcomes motivate the consideration of an extremely high number of RF-EH ICs within a single receiver unit in order to maintain the stability of the Quantum battery, as well as to ensure an optimal transmit power within the safety limits. The proposed novel system based on RF-EH ICs assisted Quantum-battery may find applications in several domains where the battery concerns are prevalent. Some examples of its prospective applications include electric vehicles, electric 
scooters, laptops, watches and many other devices, which use batteries.

\section{APPENDIX A}

\section{Closed-FORM SOLUTION FOR THE TRANSMIT POWER}

The non-linear EH constraint at end-user, as in (6), is given by

$$
N \frac{\mathcal{E}^{\prime}}{1-\phi} \cdot\left(\frac{1}{1+e^{\left(-\alpha P_{T}|h|^{2}+\alpha \beta\right)}}-\phi\right) \geq \xi,
$$

where $\xi$ is the harvested energy demand at the end-user.

The expression in (9) can be re-arranged as

$$
\frac{1}{1+e^{\left(-\alpha P_{T}|h|^{2}+\alpha \beta\right)}} \geq \xi \cdot\left(\frac{1-\phi}{N \mathcal{E}^{\prime}}\right)+\phi
$$

which can be written as

$$
1+e^{\left(-\alpha P_{T}|h|^{2}+\alpha \beta\right)} \leq \frac{N \mathcal{E}^{\prime}}{\xi(1-\phi)+\phi N \mathcal{E}^{\prime}} .
$$

The equation in (11) implies that

$$
e^{\left(-\alpha P_{T}|h|^{2}+\alpha \beta\right)} \leq \frac{N \mathcal{E}^{\prime}-\left(\xi(1-\phi)+\phi N \mathcal{E}^{\prime}\right)}{\xi(1-\phi)+\phi N \mathcal{E}^{\prime}} .
$$

Taking natural logarithm on both the sides of (12), we have

$$
-\alpha P_{T}|h|^{2}+\alpha \beta \leq \ln \left(\frac{N \mathcal{E}^{\prime}-\left(\xi(1-\phi)+\phi N \mathcal{E}^{\prime}\right)}{\xi(1-\phi)+\phi N \mathcal{E}^{\prime}}\right) .
$$

Further simplification of (13) leads to the equivalent constraint on the transmit power

$$
P_{T} \geq \frac{1}{\alpha|h|^{2}}\left(\alpha \beta-\ln \left(\frac{N \mathcal{E}^{\prime}-\xi(1-\phi)-N \phi \mathcal{E}^{\prime}}{\xi(1-\phi)+N \phi \mathcal{E}^{\prime}}\right)\right) \text {. }
$$

From (14), it is clear that in order to minimize the total transmit power, the equality in (14) must hold. Therefore, we reach to the closed-form expression for $P_{T}$, as follows.

$$
P_{T}=\frac{1}{\alpha|h|^{2}}\left(\alpha \beta-\ln \left(\frac{N \mathcal{E}^{\prime}-\xi(1-\phi)-N \phi \mathcal{E}^{\prime}}{\xi(1-\phi)+N \phi \mathcal{E}^{\prime}}\right)\right) \text {. }
$$

QED.

\section{REFERENCES}

[1] P. Botsinis, D. Alanis, Z. Babar, H. V. Nguyen, D. Chandra, S. X. Ng, and L. Hanzo, "Quantum Search Algorithms for Wireless Communications," IEEE Commun. Surveys Tuts., vol. 21, no. 2, pp. 1209-1242, 2019.

[2] F. Salek, A. Anshu, M. Hsieh, R. Jain, and J. R. Fonollosa, "One-Shot Capacity Bounds on the Simultaneous Transmission of Classical and Quantum Information," IEEE Trans. Inf. Theory, vol. 66, no. 4, pp. 2141-2164, 2020.

[3] A. S. Cacciapuoti, M. Caleffi, R. Van Meter, and L. Hanzo, "When Entanglement Meets Classical Communications: Quantum Teleportation for the Quantum Internet," IEEE Trans. Commun., vol. 68, no. 6, pp. 3808-3833, 2020.

[4] S. Gautam and et al., "Multigroup Multicast Precoding for Energy Optimization in SWIPT Systems With Heterogeneous Users," IEEE Open Journal of the Communications Society, vol. 1, pp. 92-108, 2020.

[5] S. Gautam, T. X. Vu, S. Chatzinotas, and B. Ottersten, "Cache-Aided Simultaneous Wireless Information and Power Transfer (SWIPT) With Relay Selection," IEEE J. Sel. Areas Commun., vol. 37, no. 1, pp. 187-201, Jan 2019.

[6] Ridddle, A Quantum Battery That Never Runs Out, Jan 2020, https: //www.youtube.com/watch?v=7fM4e0cx5zU.

[7] F. Campaioli, F. A. Pollock, F. C. Binder, L. Céleri, J. Goold, S. Vinjanampathy, and K. Modi, "Enhancing the Charging Power of Quantum Batteries," Physical Review Letters, vol. 118, no. 15, Apr 2017.

[8] G. M. Andolina, M. Keck, A. Mari, M. Campisi, V. Giovannetti, and M. Polini, "Extractable Work, the Role of Correlations, and Asymptotic Freedom in Quantum Batteries," Physical Review Letters, vol. 122, no. 4, Feb 2019.
[9] G. M. Andolina, D. Farina, A. Mari, V. Pellegrini, V. Giovannetti, and M. Polini, "Charger-mediated energy transfer in exactly solvable models for quantum batteries," Physical Review B, vol. 98, no. 20, Nov 2018.

[10] J. Q. Quach and W. J. Munro, "Using Dark States to Charge and Stabilize Open Quantum Batteries," Physical Review Applied, vol. 14, no. 2, Aug 2020.

[11] J. Liu, D. Segal, and G. Hanna, "Loss-Free Excitonic Quantum Battery," The Journal of Physical Chemistry C, vol. 123, no. 30, pp. 1830318314, 2019.

[12] F.-Q. Dou, Y.-J. Wang, and J.-A. Sun, "Closed-loop three-level charged quantum battery," 2020.

[13] W. Lumpkins, "Nikola Tesla's Dream Realized: Wireless power energy harvesting.," IEEE Consumer Electronics Magazine, vol. 3, no. 1, pp. 39-42, 2014.

[14] C. R. Valenta and G. D. Durgin, "Harvesting Wireless Power: Survey of Energy-Harvester Conversion Efficiency in Far-Field, Wireless Power Transfer Systems," IEEE Microwave Magazine, vol. 15, no. 4, pp. 108$120,2014$.

[15] L. Wang, P. Todaria, A. Pandey, J. O'Connor, B. Chernow, and L. Zuo, "An Electromagnetic Speed Bump Energy Harvester and Its Interactions With Vehicles," IEEE/ASME Trans. Mechatronics, vol. 21, no. 4, pp. 1985-1994, 2016.

[16] T. Ruan, Z. J. Chew, and M. Zhu, "Energy-Aware Approaches for Energy Harvesting Powered Wireless Sensor Nodes," IEEE Sensors J., vol. 17, no. 7, pp. 2165-2173, 2017.

[17] Z. Yang, W. Xu, and M. S.-Bahaei, "Energy Efficient UAV Communication With Energy Harvesting," IEEE Trans. Veh. Technol., vol. 69, no. 2, pp. 1913-1927, 2020.

[18] L. R. Varshney, "Transporting information and energy simultaneously," in IEEE Int. Symp. Inf. Theory, July 2008, pp. 1612-1616.

[19] Z. Ding, C. Zhong, D. W. K. Ng, M. Peng, H. A. Suraweera, R. Schober, and H. V. Poor, "Application of smart antenna technologies in simultaneous wireless information and power transfer," IEEE Comm. Mag., vol. 53, no. 4, pp. 86-93, April 2015.

[20] T. D. P. Perera, D. N. K. Jayakody, S. K. Sharma, S. Chatzinotas, and J. Li, "Simultaneous Wireless Information and Power Transfer (SWIPT): Recent Advances and Future Challenges," IEEE Commun. Surveys Tuts., vol. 20, no. 1, pp. 264-302, 2018.

[21] J. Kang, I. Kim, and D. I. Kim, "Joint Tx Power Allocation and Rx Power Splitting for SWIPT System With Multiple Nonlinear Energy Harvesting Circuits," IEEE Wireless Commun. Lett., vol. 8, no. 1, pp. 53-56, 2019.

[22] G. Ma, J. Xu, Y. Zeng, and M. R. V. Moghadam, "A Generic Receiver Architecture for MIMO Wireless Power Transfer With Nonlinear Energy Harvesting," IEEE Signal Process. Lett., vol. 26, no. 2, pp. 312$316,2019$.

[23] A. Y. Awan, M. Ali, M. Naeem, F. Qamar, and M. N. Sial, "Joint Network Admission Control, Mode Assignment, and Power Allocation in Energy Harvesting Aided D2D Communication," IEEE Trans. Ind. Informat., vol. 16, no. 3, pp. 1914-1923, 2019.

[24] A. N. Jordan, B. Sothmann, R. Sánchez, and M. Büttiker, "Powerful and efficient energy harvester with resonant-tunneling quantum dots," Physical Review B, vol. 87, no. 7, Feb 2013.

[25] B. Clerckx and E. Bayguzina, "Waveform Design for Wireless Power Transfer," IEEE Trans. Signal Process., vol. 64, no. 23, pp. 6313-6328, 2016.

[26] J. Guo, H. Zhang, and X. Zhu, "Theoretical analysis of RF-DC conversion efficiency for class-F rectifiers," IEEE Trans. Microw. Theory Techn., vol. 62, no. 4, pp. 977-985, 2014.

[27] E. Boshkovska, D. W. K. Ng, N. Zlatanov, and R. Schober, "Practical Non-Linear Energy Harvesting Model and Resource Allocation for SWIPT Systems," IEEE Wireless Commun. Lett., vol. 19, no. 12, pp. 2082-2085, Dec 2015.

[28] S. Gautam, E. Lagunas, S. Chatzinotas, and B. Ottersten, "Relay Selection and Resource Allocation for SWIPT in Multi-User OFDMA Systems," IEEE Trans. Wireless Commun., vol. 18, no. 5, pp. $2493-$ $2508,2019$.

[29] S. Boyd, S. P. Boyd, and L. Vandenberghe, Convex optimization, Cambridge university press, 2004.

[30] “MATLAB Optimization Toolbox (fmincon)," 9.7.0.1296695 (R2019b) Update 4, The MathWorks, Natick, MA, USA.

[31] P. Series, "Propagation data and prediction methods for the planning of short-range outdoor radiocommunication systems and radio local area networks in the frequency range $300 \mathrm{MHz}$ to $100 \mathrm{GHz}$," tech. rep., ITU, Tech. Rep. ITU-R, 2017. 